\title{
Effects of mevalonate kinase interference on cell differentiation, apoptosis, prenylation and geranylgeranylation of human keratinocytes are attenuated by farnesyl pyrophosphate or geranylgeranyl pyrophosphate
}

\author{
MIN LI ${ }^{1 *}$, WEI MIN ${ }^{1 *}$, JIANBO WANG $^{2}, \mathrm{LU}_{\text {WANG }}{ }^{1}$, YAN LI $^{1}$, \\ NAIHUI ZHOU ${ }^{1}$, ZILIANG YANG ${ }^{1}$ and QIHONG QIAN ${ }^{1}$ \\ ${ }^{1}$ Department of Dermatology, First Affiliated Hospital of Soochow University, Suzhou, Jiangsu 215006; \\ ${ }^{2}$ Department of Dermatology, Henan Provincial People's Hospital, People's Hospital of Zhengzhou University, \\ School of Clinical Medicine, Henan University, Zhengzhou, Henan 450003, P.R. China
}

Received December 17, 2018; Accepted November 14, 2019

DOI: $10.3892 /$ etm.2020.8569

\begin{abstract}
Mevalonate kinase (MVK) mutations were previously identified in disseminated superficial actinic porokeratosis. However, the role of MVK in differentiation, apoptosis and prenylation of keratinocytes requires further investigation. Farnesyl pyrophosphate (FPP) and geranylgeranyl pyrophosphate (GGPP) of the mevalonate pathway attach to small $\mathrm{G}$ proteins, and serve as molecular switches in biochemical pathways. Therefore, the aim of the present study was to investigate the role of MVK in the expression of keratin 1 and involucrin, apoptosis, protein prenylation and the processing of small $\mathrm{G}$ proteins. HaCat human keratinocytes were transfected with viruses carrying MVK interference and overexpression vectors, respectively. The mRNA expression of MVK, keratin 1 and involucrin was detected by reverse transcription-quantitative PCR. Protein expression of MVK, keratin 1, involucrin, lamin A, HRAS, KRAS, NRAS, Rho E, Rho B, Rho A, RAC1 and cdc42 in HaCat cells was detected by western blotting. The apoptotic rates of HaCat cells and protein prenylation levels were examined by flow cytometry. The expression of MVK in HaCat cells was significantly decreased in the interference groups, and markedly increased in the overexpression group compared with the negative
\end{abstract}

Correspondence to: Dr Qihong Qian or Dr Ziliang Yang Department of Dermatology, First Affiliated Hospital of Soochow University, 188 Shizi Street, Suzhou, Jiangsu 215006, P.R. China

E-mail: qqihong@126.com

E-mail: yz16405@163.com

"Contributed equally

Key words: mevalonate kinase, farnesyl pyrophosphate, geranylgeranyl pyrophosphate, apoptosis, prenylation, small G-proteins control groups. The mRNA and protein expression levels of keratin 1 and involucrin were significantly decreased following interference of MVK expression, and the decrease was markedly attenuated by FPP. Furthermore, the apoptotic rate was markedly increased following MVK interference, and the increase was significantly attenuated by GGPP. The overexpression of MVK significantly decreased the apoptotic rate of HaCat cells. The prenylation levels after MVK interference was notably decreased, which was markedly attenuated by GGPP. The overexpression of MVK significantly increased the prenylation levels of HaCat cells. FPP or GGPP reversed MVK interference-induced decrease in geranylgeranylation levels of lamin A, HRAS, KRAS, NRAS, Rho E, Rho B, Rho A, $\mathrm{RAC1}$ and cdc42. In conclusion, MVK interference decreases the expression of differentiation markers, increases apoptosis, and decreases protein prenylation and geranylgeranylation levels in keratinocytes. These changes are attenuated by FPP or GGPP.

\section{Introduction}

The mevalonate kinase (MVK) gene has 10 coding exons and one non-coding exon at chromosome $12 \mathrm{q} 24$. The MVK protein is encoded by two transcripts of MVK and is expressed in various tissues, such as the human skin (1). MVK catalyzes the phosphorylation of mevalonic acid into 5-phosphomevalonate, and functions downstream of 3-hydroxy-3-methyl-glutaryl-coenzyme A reductase (HMG-CoA) in the mevalonate pathway. The mevalonate pathway provides crucial molecules to cells, and may be an important metabolic pathway in human skin (2). Cholesterol, which is essential in the function of skin barrier, derives from mevalonate pathway (3) and MVK plays an important role in the synthesis of isoprenoid and cholesterol. Furthermore, the mevalonate pathway was reported to regulate the gene expression of keratin (4). MVK mutations were previously identified in disseminated superficial actinic porokeratosis by exome sequencing (5). Moreover, MVK plays an important role in 
regulating calcium-induced keratinocyte differentiation and protecting keratinocytes from apoptosis induced by type A ultraviolet radiation in cultured primary keratinocytes (5).

Keratin 1 (KRT1) and involucrin (IVL) are markers of the differentiation of keratinocytes in the spinous layer and granular layer of skin, respectively. Apoptosis and dysregulation of keratinocyte differentiation was reported to be associated with the pathogenesis of porokeratosis (6). Prenylation (farnesylation and geranylgeranylation), also known as lipidation, facilitates the attachment of molecules to cell membranes; hydrophobic molecules are added to a chemical compound or protein during prenylation. The inhibition of prenylation by HMG-CoA reductase inhibitors fluvastatin or compactin was shown to decrease the bradykinin-stimulated generation of inositol 1,4,5-triphosphate in human keratinocytes (7). However, the role of MVK in the differentiation, apoptosis and prenylation of human keratinocytes requires further investigation.

Short-chain isoprenoids farnesyl pyrophosphate (FPP) and geranylgeranyl pyrophosphate (GGPP) are the intermediate products of the mevalonate pathway; these attach to small $\mathrm{G}$ proteins covalently, and serve as molecular switches in many biochemical pathways (8). Small G proteins, such as lamin A, HRAS, KRAS, NRAS, Rho E, Rho B, Rho A, Ras-related C3 botulinum toxin substrate 1 (RAC1) and cell division control protein 42 homolog (CDC42), independently hydrolyze guanosine triphosphate (GTP) in cytosol. However, it is unclear whether FPP and GGPP could rescue the downregulation of MVK expression and whether MVK expression affects the processing of small $\mathrm{G}$ proteins.

Thus, the present study aimed to investigate the role of interference and overexpression of MVK in the expression of keratin 1 and involucrin, apoptosis, protein prenylation, and the processing of small $\mathrm{G}$ proteins.

\section{Materials and methods}

Cell culture. HaCaT cells (cat. no. C01-BH; Shanghai Novobioscience, Co., Ltd.) were cultured in Dulbecco's modified Eagle's medium (DMEM) with GlutaMax ${ }^{\mathrm{TM}}$, supplemented with $10 \%$ fetal bovine serum (FBS), $100 \mathrm{IU} / \mathrm{ml}$ penicillin and $100 \mu \mathrm{g} / \mathrm{ml}$ streptomycin (Gibco; Thermo Fisher Scientific, Inc.). Cells were seeded at $1 \times 10^{5}$ cells $/ \mathrm{ml}$, and the medium was changed every other day up to $90 \%$ confluency.

Construction of recombinant plasmids and lentiviral packaging. According to gene information of $\mathrm{MVK}$ (NM_000431.3), three-pairs of oligos encoding the target sequence of MVK (Table I) and CDS domain sequences were designed, synthesized and inserted into interference vector PDS019_PL-shRNA and overexpression vector PDS159_pL6.3-CMV-GFPa1-IRES-MCS (Shanghai Novobioscience, Co., Ltd.), respectively. Interference lentiviral vectors (CL926-1-pL-shRNA-HOMO-MVK-348, CL926-2-PL-shRNA-HOMO-MVK-520, and CL926-3-pLshRNA-HOMO-MVK-1283) and overexpression lentiviral vector (CL927-pL6.3-CMV-GFPa1-IRES-homo-MVK) were produced. The negative control for interference was non-targeting sequence (5'-GAAACGATATGGGCTGAA TAC-3'), which was inserted into the vector pL-shRNA
(Novobio). The negative control for overexpression was an empty vector pL6.3-CMV-GFPa1-IRES-MCS (Novobio). Correct sequences were confirmed by Sanger sequencing. ViraPower ${ }^{\mathrm{TM}}$ Lentiviral Packaging Mix (9 $\mu \mathrm{g}$; Invitrogen; Thermo Fisher Scientific, Inc.) and the recombinant lentiviral vectors ( $3 \mu \mathrm{g}$; Invitrogen; Thermo Fisher Scientific, Inc.) were added into Opti-Minimum Essential Medium (MEM; Gibco; Thermo Fisher Scientific, Inc.) and mixed, respectively. Lipofectamine ${ }^{\circledR} 2000$ (36 $\mu$; Invitrogen; Thermo Fisher Scientific, Inc.) was mixed with Opti-MEM (1.5 ml), and incubated at room temperature for $5 \mathrm{~min}$. The plasmid solution and diluted Lipofectamine ${ }^{\circledR} 2000$ were subsequently mixed, and incubated at room temperature for $5 \mathrm{~min}$. The mixture was added into a culture dish with $293 \mathrm{~T}$ cells $\left(3 \times 10^{6}\right)$ and cells were cultured for $48 \mathrm{~h}$. Cell supernatant was collected, centrifuged at $1,500 \mathrm{x} \mathrm{g}$ for $10 \mathrm{~min}$ at room temperature and filtered. The virus solution was then condensed by centrifuging at $50,000 \times \mathrm{g}$ for $2 \mathrm{~h}$ at $4^{\circ} \mathrm{C}$, and re-suspended in DMEM. Viruses carrying MVK interference (LV542-1, LV542-2 and LV542-3, respectively) and overexpression vectors were derived, respectively. Human keratinocytes HaCaT were transfected with the viruses (multiplicity of transfection, 10) for $48 \mathrm{~h}$, and reverse transcription-quantitative PCR was utilized to detect efficiency of interference and overexpression, respectively.

$R T-q P C R$. The expression of MVK, KRT1 and IVL in HaCat cells was detected by RT-qPCR. HaCat human keratinocytes were divided into 5 groups: i) negative control; ii) MVK interference; iii) MVK interference+FPP (1 $\mu \mathrm{M})$; iv) MVK interference+GGPP (1 $\mu \mathrm{M})$; v) MVK overexpression. Prior to detecting the expression of KRT1 and IVL by RT-qPCR, $\mathrm{HaCa}$ cells were treated with $\mathrm{CaCl}_{2}(0.5 \mathrm{mM})$ for $48 \mathrm{~h}$. Total RNA was extracted from cells using TRIzol ${ }^{\circledR}$ reagent (Invitrogen; Thermo Fisher Scientific, Inc.), according to the manufacturer's protocol. SuperScript ${ }^{\mathrm{TM}}$ First-Strand Synthesis System (cat. no. 11904018; Invitrogen; Thermo Fisher Scientific, Inc.) was used for RT by applying the following temperature protocol: $65^{\circ} \mathrm{C}$ for $5 \mathrm{~min} ; 42^{\circ} \mathrm{C}$ for $60 \mathrm{~min}$ and $70^{\circ} \mathrm{C}$ for $15 \mathrm{~min}$. Each reaction mixture contained $0.5 \mu \mathrm{l}$ random primers $(0.2 \mu \mathrm{g} / \mu \mathrm{l})$ and $1 \mu \mathrm{l}$ SuperScript III reverse transcriptase (200 U/ $\mu$ l; Invitrogen; Thermo Fisher Scientific, Inc.). The specific primers used are listed in Table II. PCR was performed using a SYBR RT-qPCR mix kit (cat. no. 4309155; Invitrogen; Thermo Fisher Scientific, Inc.). The PCR conditions were as follows: Initial denaturation at $95^{\circ} \mathrm{C}$ for $2 \mathrm{~min}$, followed by 40 cycles of denaturation at $95^{\circ} \mathrm{C}$ for $10 \mathrm{sec}$, annealing at $60^{\circ} \mathrm{C}$ for $30 \mathrm{sec}$ and elongation at $70^{\circ} \mathrm{C}$ for $45 \mathrm{sec}$. PCR was performed using a CFX96 Touch $^{\mathrm{TM}}$ Real-Time PCR Detection system (Bio-Rad Laboratories, Inc.). Gene expression was determined and normalized to $\beta$-actin. The $2^{-\Delta \Delta \mathrm{Cq}}$ method was utilized to determine the relative gene expression (9).

Western blot analysis. Protein expression of MVK, keratin 1, involucrin, lamin A, HRAS, KRAS, NRAS, Rho E, Rho B, Rho A, RAC1 and CDC42 in HaCat cells was detected by western blot analysis. Prior to detecting the expression of KRT1 and IVL by western blotting, HaCat cells were treated with $\mathrm{CaCl}_{2}(0.5 \mathrm{mM})$ for $48 \mathrm{~h}$. HaCat cells were 
Table I. Oligo sequences for interference.

\begin{tabular}{ll}
\hline Oligo & \multicolumn{1}{c}{ Sequences (5' to 3') } \\
\hline MVK-348-F & CACCGCTTACCCAACATTGGTATCACGAATGATACCAATGTTGGGTAAGC \\
MVK-348-R & AAAAGCTTACCCAACATTGGTATCATTCGTGATACCAATGTTGGGTAAGC \\
MVK-520-F & CACCGCTGGCCTTTCTTTACTTATACGAATATAAGTAAAGAAAGGCCAGC \\
MVK-520-R & AAAAGCTGGCCTTTCTTTACTTATATTCGTATAAGTAAAGAAAGGCCAGC \\
MVK-1283-F & CACCGGCTTTGACTGCTTGGAAACCCGAAGGTTTCCAAGCAGTCAAAGCC \\
MVK-1283-R & AAAAGGCTTTGACTGCTTGGAAACCTTCGGGTTTCCAAGCAGTCAAAGCC \\
Non-targeting sequence & GAAACGATATGGGCTGAATAC
\end{tabular}

MVK, mevalonate kinase; F, forward; R, reverse.

Table II. Primers for reverse transcription-quantitative PCR.

\begin{tabular}{lc}
\hline Primers & \multicolumn{1}{c}{ Sequences (5' to 3') } \\
\hline MVK-F & TTCCCAGGAGCCATGTTGTC \\
MVK-R & CTTGCTCTGAGGTGGGTGTT \\
KRT1-F & TGGACAACAACCGCAGTCTC \\
KRT1-R & CTCTGGTACAAGGACTCGGC \\
IVL-F & TATTTCGGGTCCGCTAGGTG \\
IVL-R & TGAGGTTGGGATTGGGGTCA \\
Actin-F & AGGGAAATCGTGCGTGAC \\
Actin-R & CGCTCATTGCCGATAGTG
\end{tabular}

MVK, mevalonate kinase; IVL, involucrin; KRT1, keratin 1; F, forward; R, reverse.

lysed in lysis buffer (cat. no. P0013; Beyotime Institute of Biotechnology) at $4{ }^{\circ} \mathrm{C}$ with inhibitors of phosphatase and protease (cat. no. P1045; Beyotime Institute of Biotechnology). The lysis mixture was centrifuged at $4^{\circ} \mathrm{C}$ at $10,000 \mathrm{x}$ g for $10 \mathrm{~min}$, and the supernatant containing cellular proteins was utilized in following experiments. The protein concentration was measured using Bicinchoninic Acid Assay kit (Beyotime Institute of Biotechnology). Proteins were separated by SDS-PAGE on a $10 \%$ gel $(40 \mu \mathrm{g}$ per lane; at $120 \mathrm{~V})$. The separated proteins were then transferred to polyvinylidene fluoride membranes (at $100 \mathrm{~V}$ for $120 \mathrm{~min}$ ). The membranes were blocked at room temperature with $5 \%$ non-fat milk for $1 \mathrm{~h}$, and incubated with primary antibodies against MVK $(1: 1,000$; cat. no. 12228-1-AP; ProteinTech Group, Inc.), cytokeratin 1 (1:1,000; cat. no. 16848-1-AP; ProteinTech Group, Inc.), involucrin (1:1,000; cat. no. 55328-1-AP; ProteinTech Group, Inc.), lamin A (1:1,000; cat. no. 10298-1-AP; ProteinTech Group, Inc.), HRAS (1:1,000; cat. no. 18295-1-AP; ProteinTech Group, Inc.), Rho E (1:1,000; cat. no. D223036; Sangon Biotech Co., Ltd.), Rho B (1:1,000; cat. no. 14326-1-AP; ProteinTech Group, Inc.), Rho A (1:1,000; cat. no. 10749-1-AP; ProteinTech Group, Inc.), RAC1 (1:1,000; cat. no. 24072-1-AP; ProteinTech Group, Inc.) and CDC42 (1:1,000; cat. no. 10155-1-AP; ProteinTech Group, Inc.) at $4^{\circ} \mathrm{C}$ overnight, respectively. Membranes were washed with Tris-buffered saline containing Tween-20 and incubated with horseradish peroxidase-conjugated goat anti-rabbit secondary antibody (1:2,000; cat. no. D110058; Sangon Biotech Co., Ltd.) at room temperature for $1 \mathrm{~h}$. Membranes were incubated in enhanced chemiluminescence solution (cat. no. P0018A; Beyotime Institute of Biotechnology). Images were captured on film (cat. no. FF057, Beyotime Institute of Biotechnology) in the dark. Experiments were repeated three times. Blot images were quantified in greyscale using the Image-Pro Plus software (version 6.0; Media Cybernetics, Inc.).

Detection of cell apoptosis by flow cytometry. HaCat cells were radiated with UVA for $5 \mathrm{~min}$, and incubated overnight. The apoptosis of HaCat cells was detected with the Annexin V-PE/7-Aminoactinomycin D (7-AAD) apoptosis assay kit (cat. no. 40310ES20; Shanghai Yeasen Biotechnology Co., Ltd.) using flow cytometry. The cells from each group were washed twice with PBS and incubated with trypsin at $37^{\circ} \mathrm{C}$ for $1 \mathrm{~min}$. Following digestion, the cell suspension was centrifuged at $400 \mathrm{x} \mathrm{g}$ at room temperature for $5 \mathrm{~min}$. The cell pellet was resuspended with PBS and the centrifugation and resuspension steps were repeated twice. The cells were blocked with $2 \%$ bovine serum albumin (Sigma-Aldrich; Merck $\mathrm{KGaA}$ ) for $30 \mathrm{~min}$ at room temperature. 7-AAD $(5 \mu \mathrm{l})$ and Annexin V-PE $(1 \mu \mathrm{l})$ reagents were added to $100 \mu \mathrm{l}$ cell suspension, followed by incubation at room temperature for $10 \mathrm{~min}$. Cells were centrifuged at $400 \mathrm{x} \mathrm{g}$ at room temperature for $5 \mathrm{~min}$ and re-suspended with PBS three times. Cell fluorescence was detected by flow cytometry. Data were acquired on an LSRII flow cytometer (BD Biosciences) and analyzed with FlowJo software (version 10.2.64; FlowJo LLC). Annexin $\mathrm{V}^{+}$ cells were calculated in each experimental group. Apoptotic rate was defined as percentage of Annexin $\mathrm{V}^{+}$cells in each group. Experiments were performed for a total of three times.

Detection of protein prenylation by flow cytometry. The protein prenylation levels of HaCat cells were detected using flow cytometry. The cells from each group were washed with PBS twice and incubated with trypsin at $37^{\circ} \mathrm{C}$ for $1 \mathrm{~min}$. Following digestion, the cell suspension was centrifuged at $400 \mathrm{x} \mathrm{g}$ at room temperature for $5 \mathrm{~min}$. The cell pellet was resuspended with PBS and the centrifugation and resuspension steps were repeated twice. The cells were blocked at room temperature with $2 \%$ bovine serum albumin (Sigma-Aldrich; Merck KGaA) overnight. Anti-farnesylation antibody (1:1,000; 
cat. no. ab199481; Abcam) was added to $100 \mu \mathrm{l}$ cell suspension, followed by incubation at room temperature for $2 \mathrm{~h}$. Cells were then incubated with iFluor goat anti-rabbit $\operatorname{IgG}(\mathrm{H}+\mathrm{L})(1: 2,000$; cat. no. 16803; AAT Bioquest Inc.) at room temperature for $1 \mathrm{~h}$. Cells were centrifuged at $400 \mathrm{x} \mathrm{g}$ at room temperature for 5 min and resuspended with PBS three times. Cell fluorescence was then detected by flow cytometry. Data were acquired on an LSRII flow cytometer (BD Biosciences,) and analyzed with FlowJo software. Cells with positive staining were calculated in each experimental group. Experiments were performed for a total of three times.

Statistical analysis. Statistical data were analyzed by GraphPad Prism version 5.0 software (GraphPad Software, Inc.). The results are presented as mean \pm standard deviation (SD). Differences among $\geq 3$ groups were compared by one-way analysis of variance followed by the Bonferroni post-hoc test. $\mathrm{P}<0.05$ was considered to indicate a statistically significant difference.

\section{Results}

Confirmation of interference and overexpression of MVK in $\mathrm{HaCat}$ cells. Following transfection, the mRNA expression of MVK in HaCat cells was significantly decreased in LV542-1 (MVK-348) and LV542-3 (MVK-1283) groups compared with the negative control group $(\mathrm{P}<0.05$; Fig. 1). In addition, the mRNA expression of MVK in HaCat cells was markedly increased in the overexpression group compared with the negative control group ( $\mathrm{P}<0.001$; Fig. 1A). Although there was no statistical significance in the relative mRNA expression of MVK between LV542-1 and LV542-3 groups, the mean value was lower in LV542-3 group (0.5, LV542-1 group; and 0.49, LV542-3 group). Thus, LV542-3 was used for interference of MVK in subsequent experiments. Moreover, the protein expression of MVK was markedly decreased in the interference groups, and increased in the overexpression group, compared with the negative control groups $(\mathrm{P}<0.001$ for all groups; Fig. 1B). These research findings indicate that the interference and overexpression in HaCat cells were successfully induced.

Decreased expression of keratin 1 and involucrin following MVK interference was markedly attenuated by FPP. Compared with the negative control group, the mRNA and protein expression of keratin 1 and involucrin were significantly decreased following interference of MVK expression $(\mathrm{P}<0.01$; Fig. 2). The decrease in the expression of keratin 1 and involucrin following MVK interference was markedly attenuated by FPP $(\mathrm{P}<0.01$; Fig. 2). GGPP was not shown to restore the expression of keratin 1 and involucrin following MVK interference. On the other hand, the overexpression of MVK did not alter the expression of keratin 1 and involucrin.

Increase in apoptotic rate following MVK interference was significantly attenuated by GGPP. The apoptotic rate following MVK interference was markedly increased compared with the negative control group $(\mathrm{P}<0.01$; Fig. 3$)$. The increase in apoptotic rate following MVK interference was significantly attenuated by GGPP ( $<<0.001$; Fig. 3$)$, but not by FPP. The overexpression of MVK significantly decreased the apoptotic rate of HaCat cells $(\mathrm{P}<0.01$; Fig. 3$)$.

Decrease in protein prenylation levels following MVK interference was markedly attenuated by GGPP. The protein prenylation levels following MVK interference was notably decreased compared with the negative control group $(\mathrm{P}<0.01$; Fig. 4). The decrease in prenylation levels following $M V K$ interference was notably attenuated by GGPP (P<0.05; Fig. 4), but not by FPP. The overexpression of MVK significantly increased the prenylation levels of HaCat cells $(\mathrm{P}<0.001$; Fig. 4).

FPP or GGPP reversed MVK interference-induced decrease in geranylgeranylation levels of small $G$ proteins. To examine the geranylgeranylation levels of small $\mathrm{G}$ proteins in $\mathrm{HaCat}$ cells following interference of MVK, the presence of processed (geranylated) and unprocessed (not geranylated) forms of proteins were evaluated (10). Geranylated lamin A, HRAS, KRAS, NRAS, Rho E, Rho B, Rho A, RAC1 and cdc42 decreased in HaCat cells following the interference of MVK (Fig. 5). Furthermore, FPP reversed MVK interference-induced decrease in geranylgeranylation levels of Lamin A, HRAS and Rho E. In addition, GGPP reversed MVK interference-induced decrease in geranylgeranylation levels of Rho A, RAC1 and cdc42. Both FPP and GGPP reversed MVK interference-induced decrease in geranylgeranylation levels of KRAS, NRAS and Rho B (Fig. 5). Moreover, the overexpression of MVK increased the geranylgeranylation levels of Lamin A, HRAS, KRAS, NRAS, Rho E, Rho B, Rho A, RAC1 and cdc42 (Fig. 5).

\section{Discussion}

The present study demonstrated the decreased expression of keratin 1 and involucrin, following MVK interference, to be notably attenuated by FPP. In addition, the increase in apoptosis and decrease in protein prenylation following MVK interference was significantly attenuated by GGPP. The overexpression of MVK significantly decreased the apoptotic rate and increased prenylation levels. The decrease in geranylgeranylation levels of Lamin A, HRAS, KRAS, NRAS, Rho E, Rho B, Rho A, RAC1 and cdc42 was attenuated by FPP or GGPP.

The decrease in expression of keratin 1 and involucrin following MVK interference was notably attenuated by FPP. The modulation of keratin 1 and involucrin expression was shown to involved in the response of human keratinocytes to ultraviolet radiation (11). Keratin and involucrin were expressed in skin diseases such as discoid lupus erythematosus and lichen planus (12). High-cell-density phorbol ester and retinoic acid upregulated involucrin in autocrine cultures of human epidermal keratinocytes (13). Keratin and involucrin were expressed in keratoacanthoma, which might aid in diagnosis (14). The knockdown of protein kinase D1 in normal human epidermal keratinocytes also increased the mRNA expression of involucrin (15). In addition, FPP was shown to be the skin metabolite that regulated epidermal responses to inflammation, oxidative stress and migration. The cytoprotective transcriptional factor $\mathrm{Nrf} 2$ and its target genes were induced by increased levels of FPP. FPP also functioned as 
A
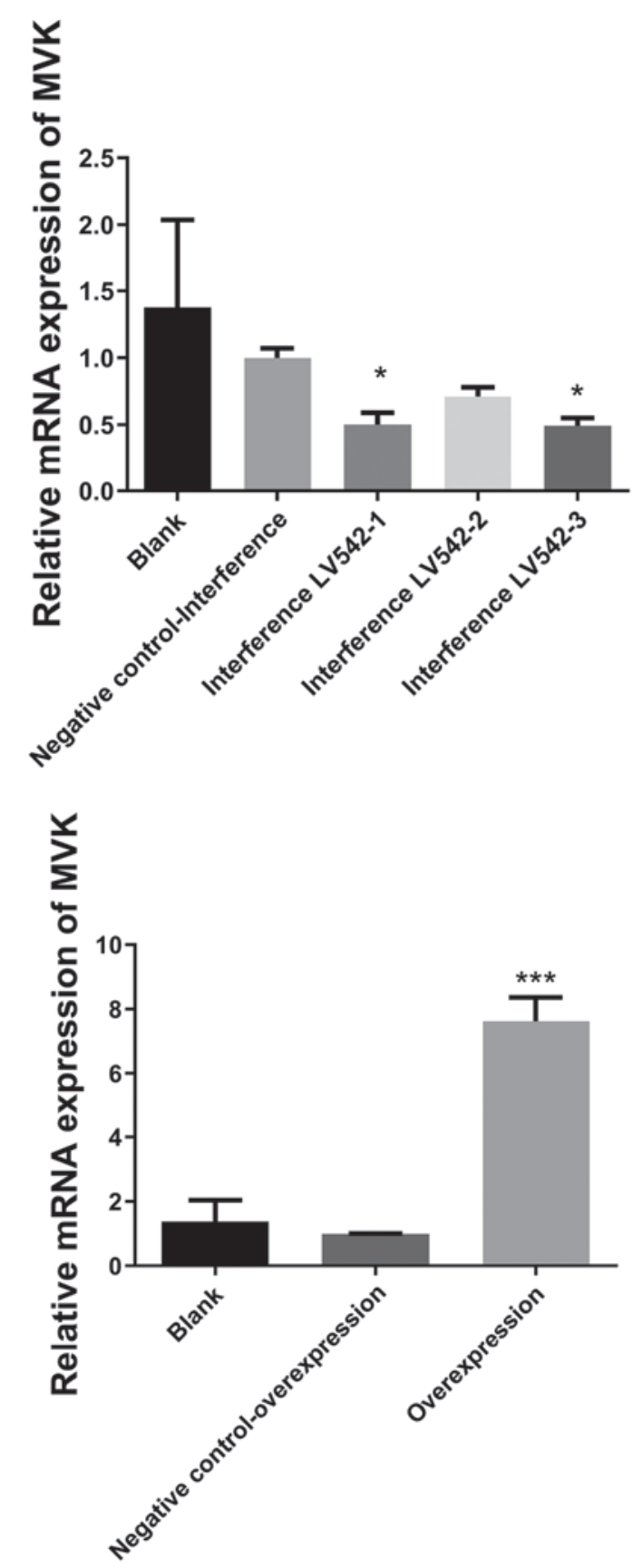

B
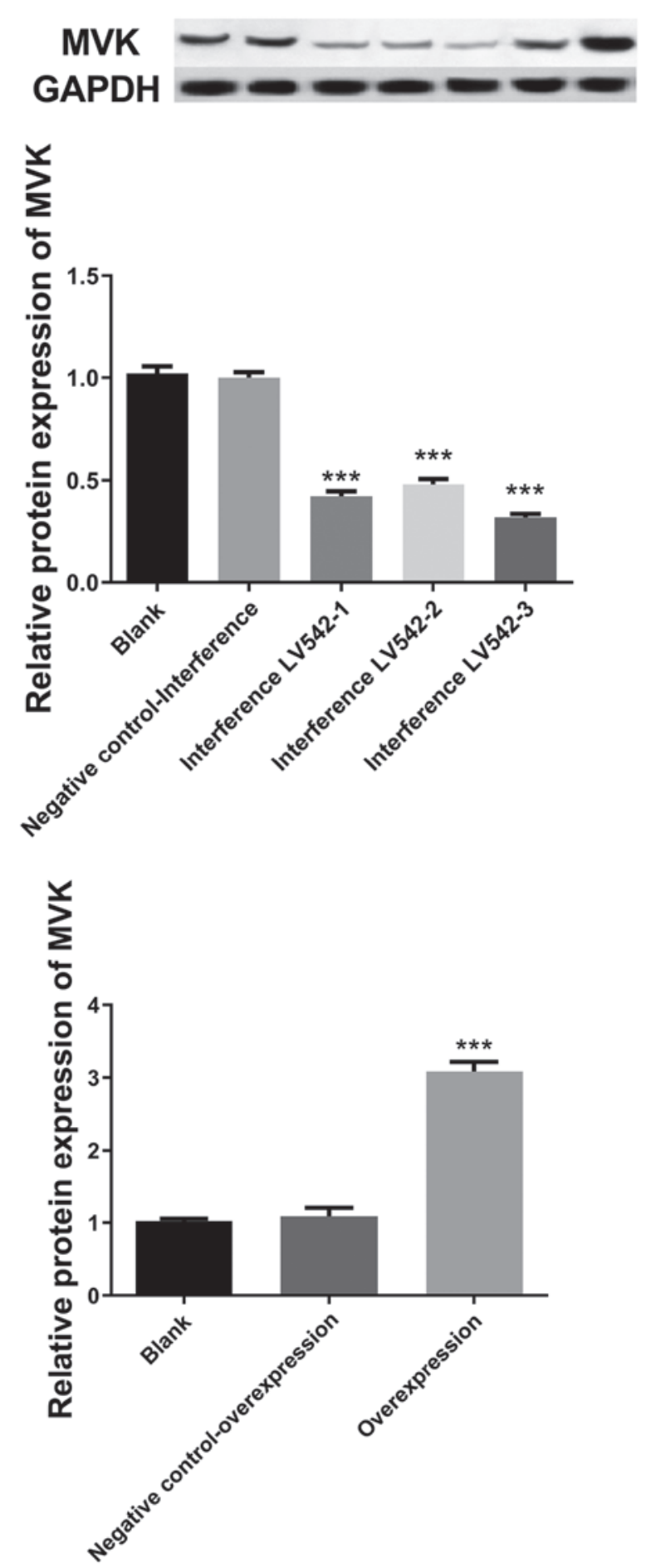

Figure 1. Interference and overexpression of MVK. (A) mRNA expression and (B) protein expression of MVK in HaCat human keratinocytes transfected with viruses carrying MVK interference and overexpression vectors, respectively. Relative mRNA expression of MVK was detected by RT-qPCR. The mRNA expression of MVK in HaCat cells was significantly decreased in LV542-1 and LV542-3 groups, and markedly increased in overexpression group compared with the negative control groups. The protein expression of MVK was markedly decreased in interference groups, and increased in overexpression group compared with the negative control groups. Data presented as mean $\pm \mathrm{SD}, \mathrm{n}=3$ /group. ${ }^{*} \mathrm{P}<0.05$ and ${ }^{* * * *} \mathrm{P}<0.001$ vs. negative control groups. MVK, mevalonate kinase; RT-qPCR, reverse transcription-quantitative PCR.

a ligand for glucocorticoid receptor (GR), which is a major regulator of epidermal homeostasis. Comparative microarray analyses demonstrated significant but incomplete overlap between glucocorticoid and FPP regulated genes (16). These findings suggest that FPP might have wider transcriptional impact. It is likely that FPP has transcriptional impact on the expression of differentiation markers in keratinocytes, such as keratin 1 and involucrin. The aforementioned effects of FPP were not seen in GGPP, which might explain why the expression of keratin 1 and involucrin following MVK interference 

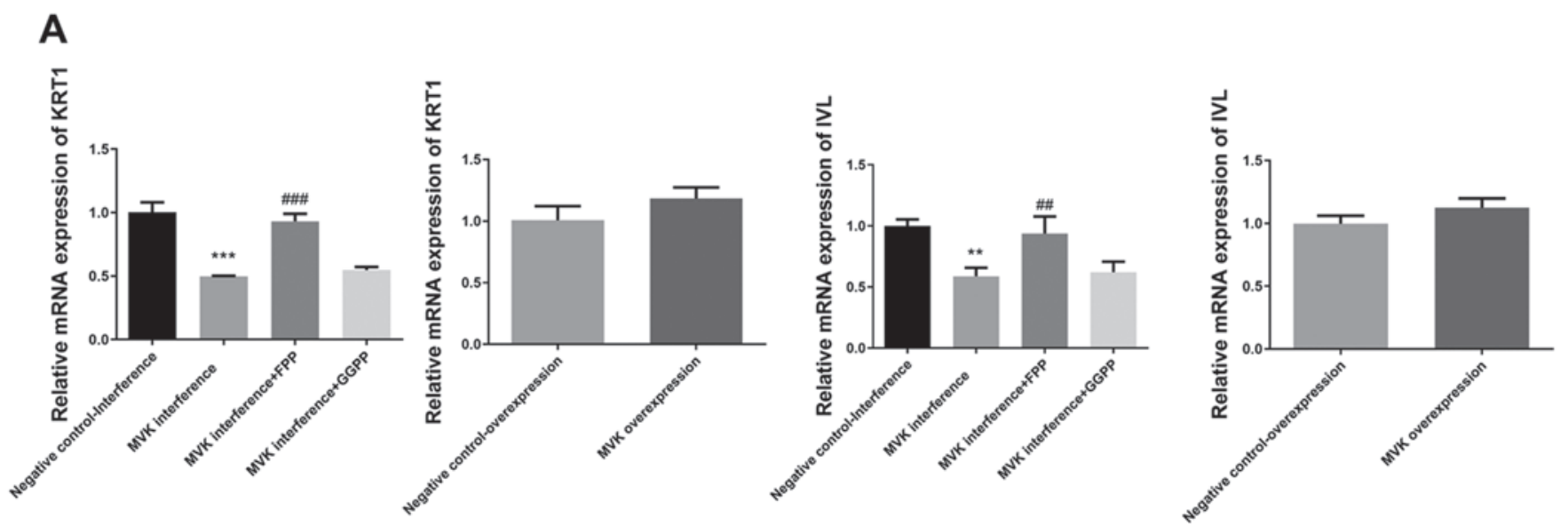

B

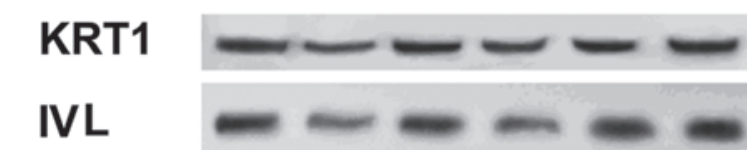

\section{GAPDH}
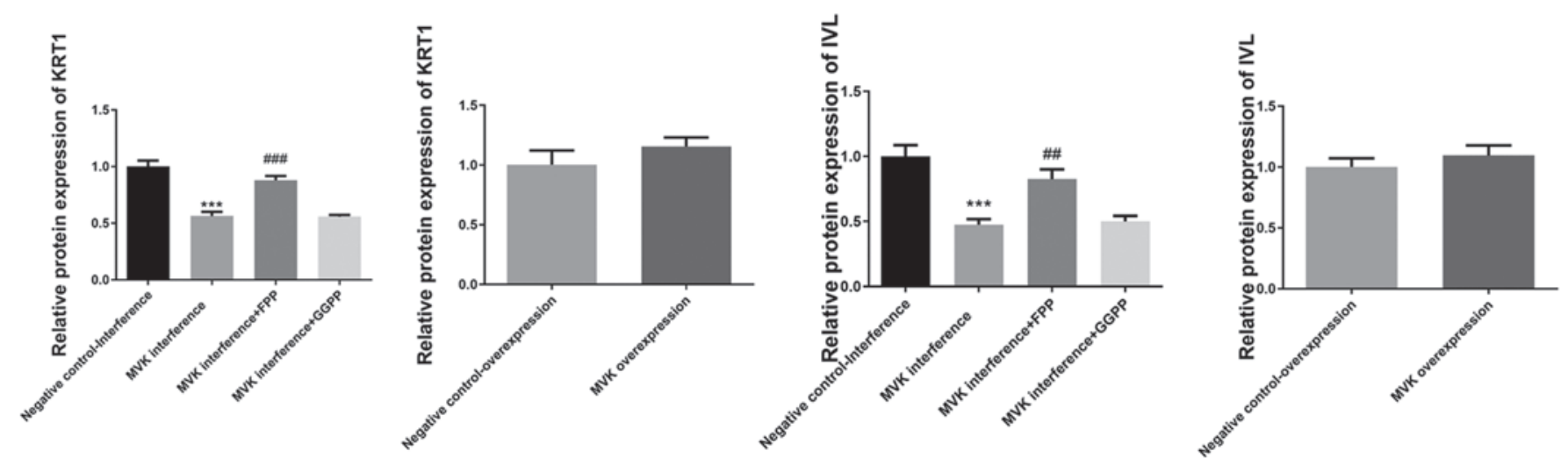

Figure 2. The mRNA and protein expression of keratin 1 and involucrin following the interference and overexpression of MVK. (A) The mRNA expression of keratin 1 and involucrin. (B) The protein expression of keratin 1 and involucirn. Relative mRNA and protein expression of keratin 1 and involucrin were detected by RT-qPCR and western blotting, respectively. Compared with the negative control group, the mRNA and protein expression of keratin 1 and involucrin were significantly decreased following interference of MVK expression. The decrease in expression of keratin 1 and involucrin following MVK interference was notably attenuated by FPP. Data presented as mean $\pm \mathrm{SD}, \mathrm{n}=3$ /group. ${ }^{* *} \mathrm{P}<0.01$ and ${ }^{* * * *} \mathrm{P}<0.001$ vs. negative control group. ${ }^{\# \#} \mathrm{P}<0.01$ and

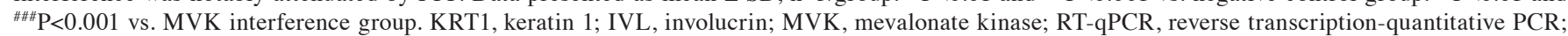
FPP, farnesyl pyrophosphate.

was not restored by GGPP. Studies on the effect of MVK on the differentiation of keratinocytes are scarce. The present study indicates that MVK is essential in keratinocyte differentiation.

In addition, the increase in apoptosis following MVK interference was significantly attenuated by GGPP, whereas the overexpression of MVK significantly decreased the apoptotic rate of human keratinocytes. Autophagy impairment, apoptosis, and lack of prenylated proteins were observed in SH-SY5Y neuronal cell model of MVK deficiency (17). In addition, MVK mutation and deficiency were shown to cause a rare autosomal recessive disease called mevalonic aciduria. Patients with mevalonic aciduria had recurrent fever episodes with severe neurologic impairments or death in early childhood. The neurodegeneration in patients with mevalonic aciduria might be associated with both the mitochondria-mediated intrinsic apoptosis pathway (caspase 9 and 3) and pyroptosis (caspase 1) (18). In addition, statins were reported to induce apoptosis in glioblastoma when the biosynthesis of GGPP was inhibited and consequently decreased levels of Akt and phosphorylated extracellular signal-regulated kinase 1/2 (ERK1/2) (19). Therefore, GGPP might inhibit apoptosis by modulating Akt and ERK. The aforementioned effect of GGPP was not evident in FPP, which may explain the reason for the increase in apoptotic rate following MVK interference not being significantly attenuated by FPP. Further investigation is required to elucidate the detailed molecular mechanisms. 
A
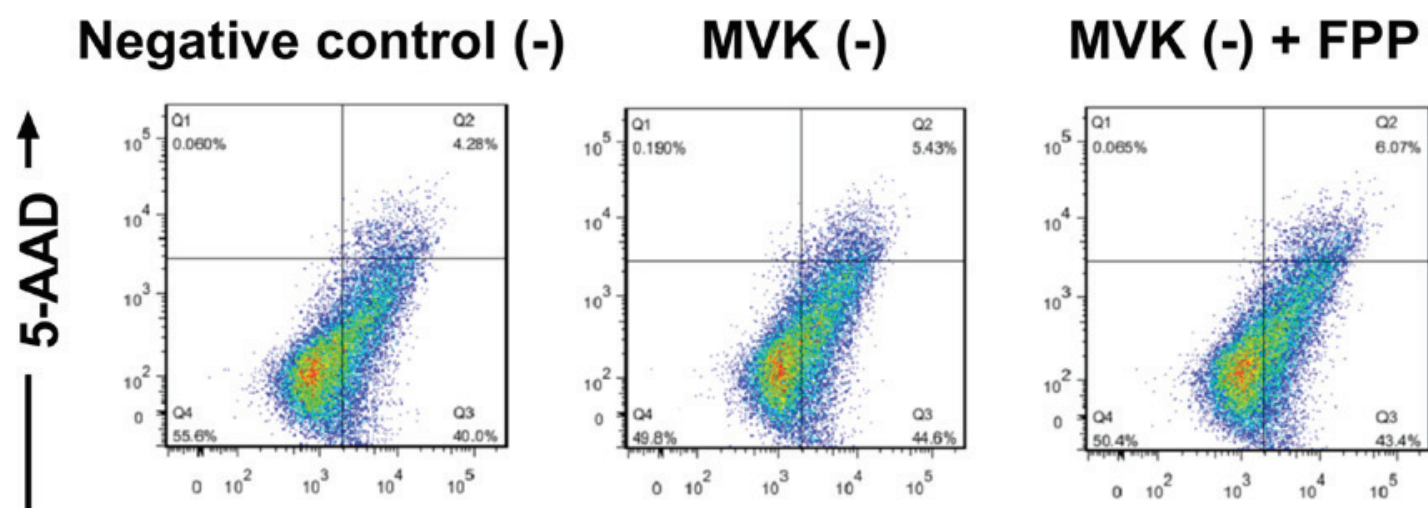

\section{MVK (-) + GGPP}
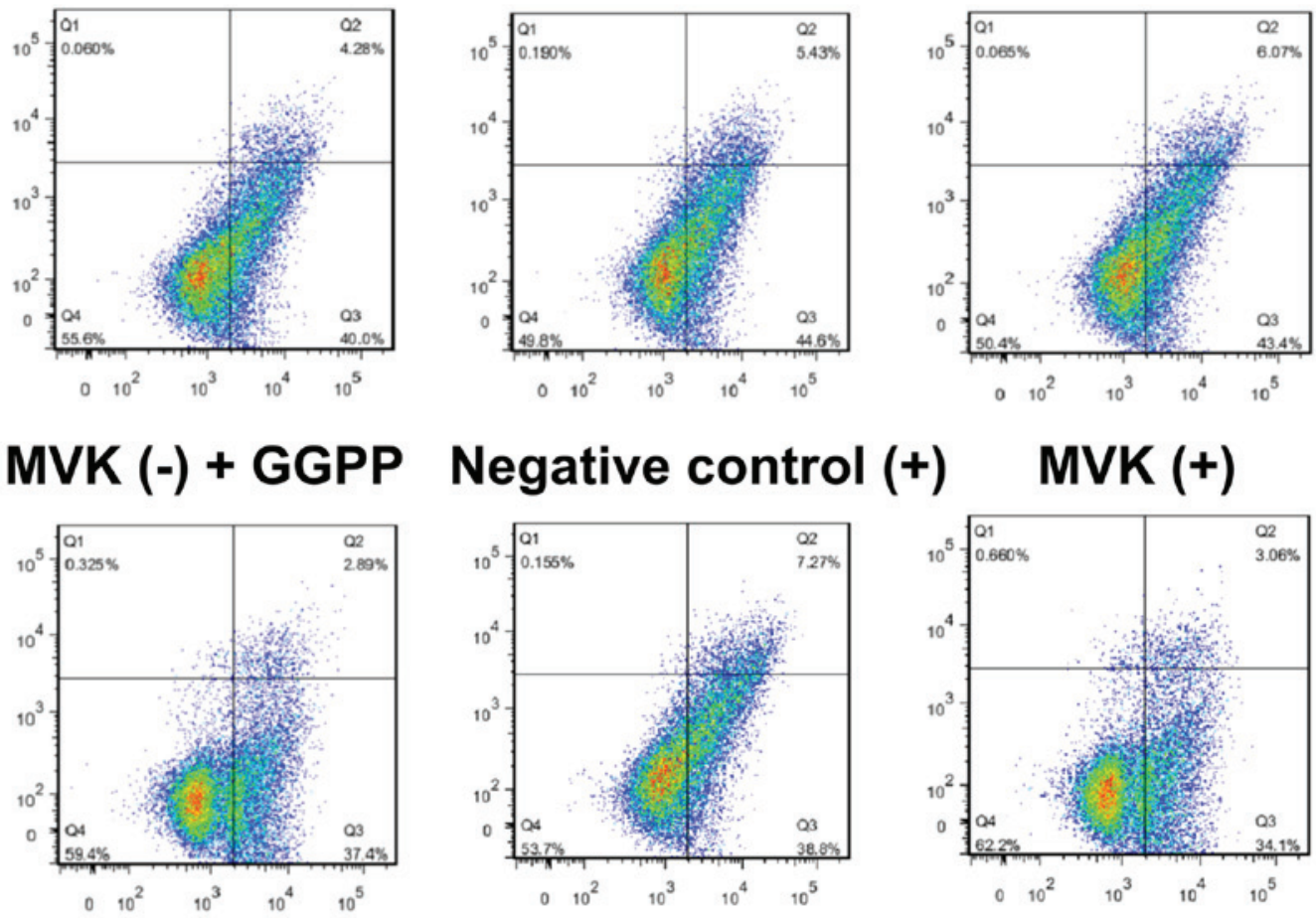

Annexin V

B
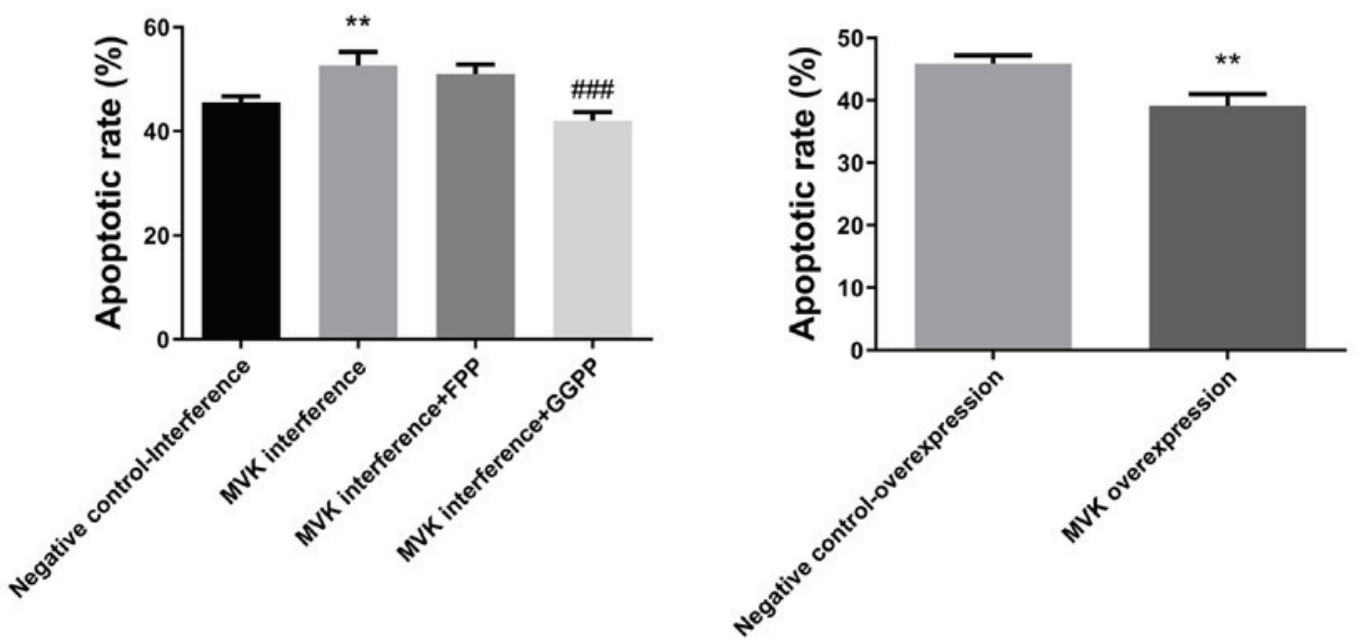

Figure 3. Cell apoptosis following the interference and overexpression of MVK. (A) Representative plot images of flow cytometry. (B) Statistical analysis of apoptotic rates. The apoptotic rates of HaCat cells were examined by flow cytometry; apoptotic cells were Annexin $\mathrm{V}^{+}$. The apoptotic rate in MVK interference group was markedly increased compared with the negative control group. The increase in apoptotic rate following MVK interference was significantly attenuated by GGPP. The overexpression of MVK significantly decreased the apoptotic rate of HaCat cells. Data presented as mean $\pm \mathrm{SD}, \mathrm{n}=3 / \mathrm{group} .{ }^{* * *} \mathrm{P}<0.01$ vs. negative control groups. ${ }^{\# \# / P} \mathrm{P}<0.001$ vs. MVK interference group. (-), interference. (+), overexpression. MVK, mevalonate kinase; GGPP, geranylgeranyl pyrophosphate; 7-AAD, 7-Aminoactinomycin D; FPP, farnesyl pyrophosphate.

The present study revealed decreased protein prenylation level following MVK interference to be significantly attenuated by GGPP. The overexpression of MVK significantly increased the prenylation levels in human keratinocytes. Mutations in
MVK were reported to result in temperature-induced defect in the prenylation of small $\mathrm{G}$ proteins in lymphoblast cell lines (20). Furthermore, defective protein prenylation was shown to be a diagnostic biomarker of MVK deficiency (21). 

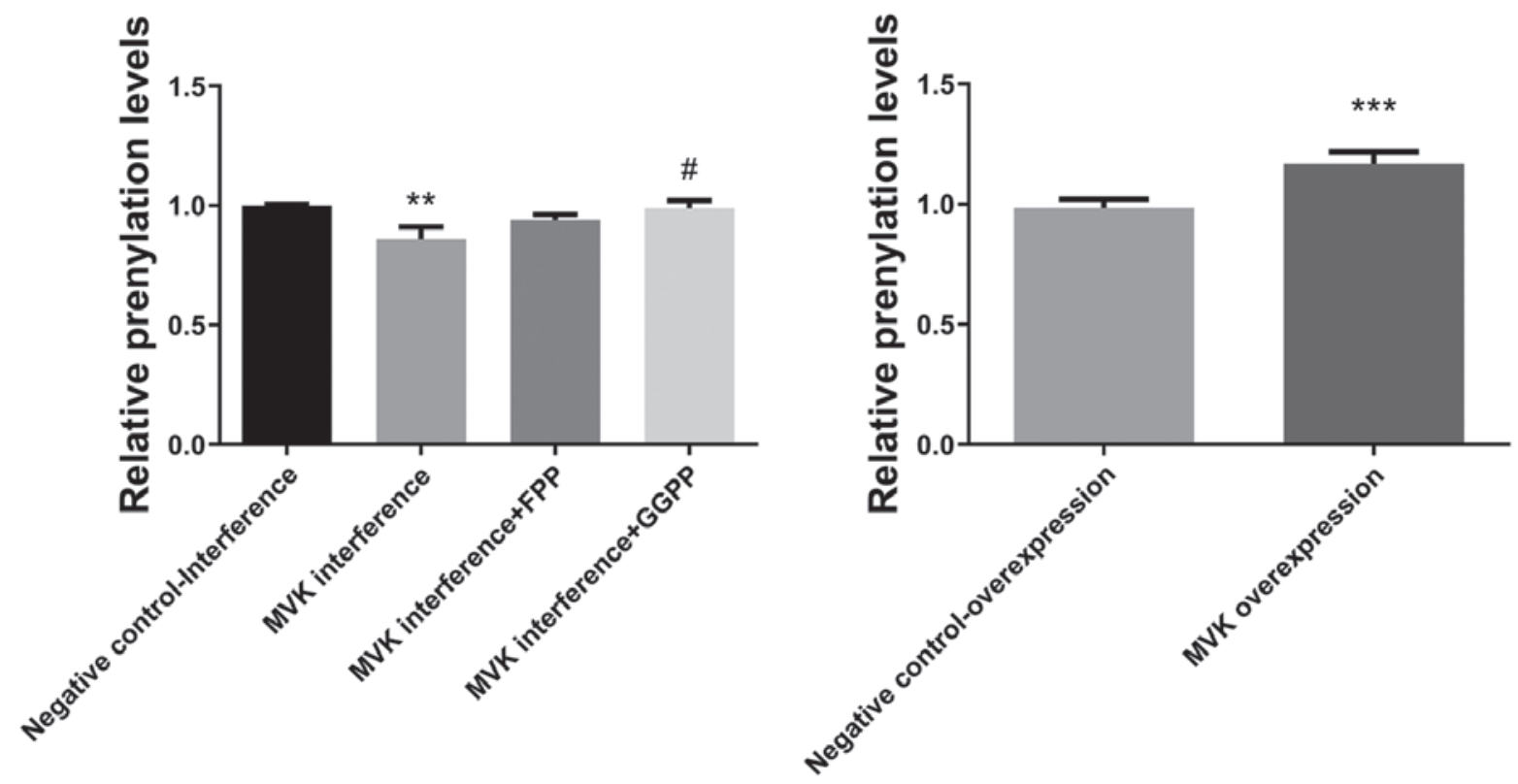

Figure 4. Protein prenylation levels following the interference and overexpression of MVK. Protein prenylation levels were examined by flow cytometry. The prenylation levels following MVK interference was notably decreased compared with the negative control group. The decrease in prenylation levels following MVK interference was notably attenuated by GGPP. The overexpression of MVK significantly increased prenylation levels of HaCat cells. Data presented as mean $\pm \mathrm{SD}, \mathrm{n}=3$ /group. ${ }^{* *} \mathrm{P}<0.01$ and ${ }^{* * * *} \mathrm{P}<0.001$ vs. negative control groups. ${ }^{*} \mathrm{P}<0.05$ vs. MVK interference group. MVK, mevalonate kinase; GGPP, geranylgeranyl pyrophosphate; FPP, farnesyl pyrophosphate.

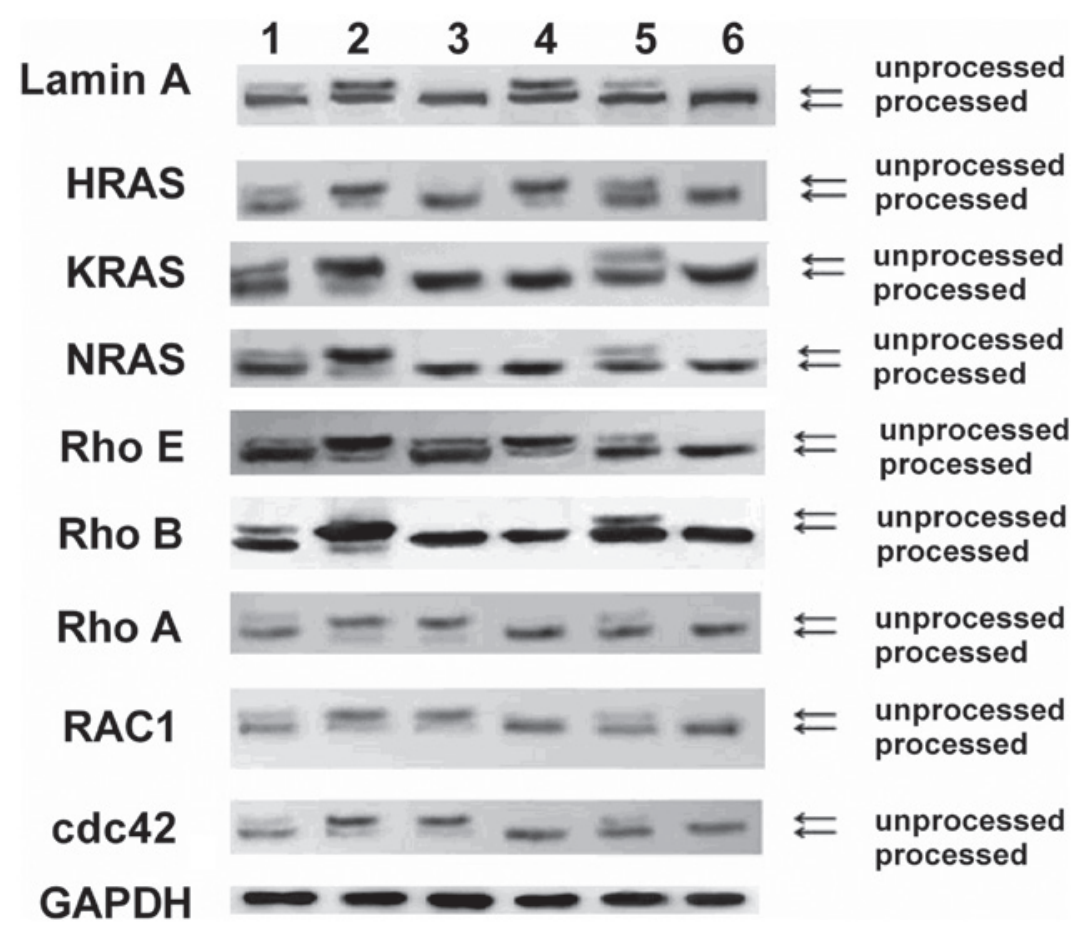

Figure 5. Geranylgeranylation levels of small G proteins following the interference and overexpression of MVK. The geranylgeranylated lamin A, HRAS, KRAS, NRAS, Rho E, Rho B, Rho A, RAC1 and cdc42 decreased in HaCat cells following interference of MVK. FPP reversed MVK interference-induced decrease in geranylgeranylation levels of lamin A, HRAS and Rho E. In addition, GGPP reversed MVK interference-induced decrease in geranylgeranylation levels of Rho A, RAC1 and cdc42. Both FPP and GGPP reversed MVK interference-induced decrease in geranylgeranylation levels of KRAS, NRAS and Rho B. 1, negative control-interference; 2, MVK interference; 3, MVK interference+FPP, 4, MVK interference+GGPP; 5, negative control-overexpression; 6, MVK overexpression. MVK, mevalonate kinase; GGPP, geranylgeranyl pyrophosphate; FPP, farnesyl pyrophosphate.

Moreover, thienopyrimidine-based bisphosphonate inhibitors of GGPP were shown to block protein prenylation and subsequently lead to cellular apoptosis in multiple myeloma cells (22). Hence, the decrease in protein prenylation might be another explanation for the increase in apoptosis following MVK interference. 
The decrease in geranylgeranylation levels of small G proteins, such as lamin A, HRAS, Rho E, Rho B, Rho A, RAC1 and cdc42, was attenuated by FPP or GGPP in keratinocytes. The geranygeranylation of small $\mathrm{G}$ proteins is involved in various biological processes. HMG-CoA reductase inhibitors were shown to block the activation of calcium-dependent tyrosine kinase Pyk2 by geranylgeranylation of small $\mathrm{G}$ protein Rap1 in vascular endothelial cells (23). The inhibition of geranylgeranylation was also reported to decrease angiotensin II-mediated free radical production in vascular smooth muscle cells, and small G protein Racl was involved in this process (24). In addition, geranylgeranylation determined the Rho migratory function in $\mathrm{T}$ cells (25). Transendothelial migration and invasion of human breast cancer cells were inhibited through prevention of geranylgeranylation of Rho (26). Moreover, GGPP-mediated protein geranylgeranylation was important for establishment of communication between oocyte and granulose cells, and transition from primary to secondary follicle in mouse ovary (27). GGPP-dependent plasma membrane localization of the small $\mathrm{G}$ protein RhoA was shown to be required for RhoA-mediated oncogenic signaling (28). Geranylgeranyl and farnesyl groups were attached to $\mathrm{C}$ termini of eukaryotic cell proteins by protein geranylgeranyltransferase-I (PGGT-I) and protein farnesyltransferase (PFT), respectively. Both geranylgeranyl and farnesyl groups from GGPP and FPP were transferred to their peptide or protein prenyl acceptor substrates by PGGT-I and PFT (29). It is likely that geranylgeranyl groups from GGPP and FPP were transferred to prenyl acceptor substrates of Lamin A, HRAS, Rho E, Rho B, Rho A, RAC1 and cdc42 by PGGT-I and PFT in keratinocytes. The present study revealed for the first time that FPP or GGPP attenuated the decrease in geranylgeranylation of small $\mathrm{G}$ proteins in human keratinocytes.

In conclusion, the present study demonstrated that the decrease in expression of keratin 1 and involucrin following MVK interference was notably attenuated by FPP. In addition, the increase in apoptosis and decrease in protein prenylation following MVK interference was significantly attenuated by GGPP. The decrease in geranylgeranylation levels of lamin A, HRAS, KRAS, NRAS, Rho E, Rho B, Rho A, RAC1 and cdc42 was attenuated by FPP or GGPP. Mutations in MVK may interrupt the biological function of keratinocytes and cause disseminated superficial actinic porokeratosis, by affecting protein prenylation in mevalonate pathway and small $\mathrm{G}$ proteins. Although further investigation is required to shine light on the molecular mechanisms, the present study might pave the foundation for future therapeutic strategies for diseases with MVK mutation, such as disseminated superficial actinic porokeratosis.

\section{Acknowledgements}

Not applicable.

\section{Funding}

The research was supported by the project of youth fund of the National Natural Science Foundation of China (grant nos. 31401071 and 81703144).

\section{Availability of data and materials}

All data generated or analyzed during the present study are included in this published article.

\section{Authors' contributions}

ML and NHZ conceived this study and obtained financial support. ZLY and QHQ participated in the design of the study. JBW performed data analyses. LW, YL, WM and ML performed the cell culture, protein expression studies, gene sequencing, protein blot analysis and other function studies. ML and WM wrote and revised the manuscript. All authors approved the final version of the manuscript.

\section{Ethics approval and consent to participate}

Not applicable.

\section{Patient consent for publication}

Not applicable.

\section{Competing interests}

The authors declare that they have no competing interests.

\section{References}

1. Fu Z, Wang M, Potter D, Miziorko HM and Kim JJ: The structure of a binary complex between a mammalian mevalonate kinase and ATP: Insights into the reaction mechanism and human inherited disease. J Biol Chem 277: 18134-18142, 2002.

2. Houten SM, Wanders RJ and Waterham HR: Biochemical and genetic aspects of mevalonate kinase and its deficiency. Biochim Biophys Acta 1529: 19-32, 2000.

3. Bouwstra JA and Ponec M: The skin barrier in healthy and diseased state. Biochim Biophys Acta 1758: 2080-2095, 2006.

4. Zhao Y, Gartner U, Smith FJ and McLean WH: Statins downregulate K6a promoter activity: A possible therapeutic avenue for pachyonychia congenita. J Invest Dermatol 131: 1045-1052, 2011.

5. Zhang SQ, Jiang T, Li M, Zhang X, Ren YQ, Wei SC, Sun LD, Cheng H, Li Y, Yin XY, et al: Exome sequencing identifies MVK mutations in disseminated superficial actinic porokeratosis. Nat Genet 44: 1156-1160, 2012.

6. Shen CS, Tabata K, Matsuki M, Goto T, Yokochi T and Yamanishi K: Premature apoptosis of keratinocytes and the dysregulation of keratinization in porokeratosis. $\mathrm{Br}$ J Dermatol 147: 498-502, 2002.

7. Alaei P, MacNulty EE and Ryder NS: Inhibition of protein prenylation down-regulates signalling by inflammatory mediators in human keratinocytes. Biochem Biophys Res Commun 222: 133-138, 1996.

8. McTaggart SJ: Isoprenylated proteins. Cell Mol Life Sci 63: 255-267, 2006.

9. Livak KJ and Schmittgen TD: Analysis of relative gene expression data using real-time quantitative PCR and the 2(-Delta Delta C(T)) method. Methods 25: 402-408, 2001.

10. Xia Z, Tan MM, Wong WW, Dimitroulakos J, Minden MD and Penn LZ: Blocking protein geranylgeranylation is essential for lovastatin-induced apoptosis of human acute myeloid leukemia cells. Leukemia 15: 1398-1407, 2001.

11. Moravcová M, Libra A, Dvořáková J, Víšková A, Muthný T, Velebný V and Kubala L: Modulation of keratin 1, 10 and involucrin expression as part of the complex response of the human keratinocyte cell line $\mathrm{HaCaT}$ to ultraviolet radiation. Interdiscip Toxicol 6: 203-208, 2013.

12. Ichikawa E, Watanabe $\mathrm{S}$ and Takahashi H: Keratin and involucrin expression in discoid lupus erythematosus and lichen planus. Arch Dermatol Res 289: 519-526, 1997. 
13. Poumay Y, Herphelin F, Smits P, De Potter IY and Pittelkow MR High-cell-density phorbol ester and retinoic acid upregulate involucrin and downregulate suprabasal keratin 10 in autocrine cultures of human epidermal keratinocytes. Mol Cell Biol Res Commun 2: 138-144, 1999.

14. Ichikawa E, Ohnishi T and Watanabe S: Expression of keratin and involucrin in keratoacanthoma: An immunohistochemical aid to diagnosis. J Dermatol Sci 34: 115-117, 2004.

15. Ivanova P, Atanasova G, Poumay Y and Mitev V: Knockdown of PKD1 in normal human epidermal keratinocytes increases mRNA expression of keratin 10 and involucrin: Early markers of keratinocyte differentiation. Arch Dermatol Res 300: 139-145, 2008.

16. Pastar I, Stojadinovic O, Sawaya AP, Stone RC, Lindley LE, Ojeh N, Vukelic S, Samuels HH and Tomic-Canic M: Skin metabolite, farnesyl pyrophosphate, regulates epidermal response to inflammation, oxidative stress, and migration. J Cell Physiol 231: 2452-2463, 2016.

17. Tricarico PM, Romeo A, Gratton R, Crovella S and Celsi F: Lack of prenylated proteins, autophagy impairment and apoptosis in SH-SY5Y neuronal cell model of mevalonate kinase deficiency. Cell Physiol Biochem 41: 1649-1660, 2017.

18. Tricarico PM, Marcuzzi A, Piscianz E, Monasta L, Crovella S and Kleiner G: Mevalonate kinase deficiency and neuroinflammation: Balance between apoptosis and pyroptosis. Int J Mol Sci 14: 23274-23288, 2013.

19. Yanae M, Tsubaki M, Satou T, Itoh T, Imano M, Yamazoe $Y$ and Nishida S: Statin-induced apoptosis via the suppression of ERK1/2 and Akt activation by inhibition of the geranylgeranyl-pyrophosphate biosynthesis in glioblastoma. J Exp Clin Cancer Res 30: 74, 2011.

20. Jurczyluk J, Munoz MA, Skinner OP, Chai RC, Ali N, Palendira U, Quinn JM, Preston A, Tangye SG, Brown AJ, et al: Mevalonate kinase deficiency leads to decreased prenylation of Rab GTPases. Immunol Cell Biol 94: 994-999, 2016.

21. Munoz MA, Jurczyluk J, Mehr S, Chai RC, Arts RJW, Sheu A, McMahon C, Center JR, Singh-Grewal D, Chaitow J, et al: Defective protein prenylation is a diagnostic biomarker of mevalonate kinase deficiency. J Allergy Clin Immunol 140: 873-875 e876, 2017

22. Lacbay CM, Waller DD, Park J, Gómez Palou M, Vincent F, Huang XF, Ta V, Berghuis AM, Sebag M and Tsantrizos YS: Unraveling the Prenylation-cancer paradox in multiple myeloma with novel geranylgeranyl pyrophosphate synthase (GGPPS) inhibitors. J Med Chem 61: 6904-6917, 2018
23. Satoh K, Ichihara K, Landon EJ, Inagami $\mathrm{T}$ and Tang $\mathrm{H}$ : 3-Hydroxy-3-methylglutaryl-CoA reductase inhibitors block calcium-dependent tyrosine kinase Pyk2 activation by angiotensin II in vascular endothelial cells. involvement of geranylgeranylation of small G protein Rap1. J Biol Chem 276: 15761-15767, 2001.

24. Wassmann S, Laufs U, Bäumer AT, Müller K, Konkol C, Sauer H, Böhm M and Nickenig G: Inhibition of geranylgeranylation reduces angiotensin II-mediated free radical production in vascular smooth muscle cells: Involvement of angiotensin AT1 receptor expression and Rac1 GTPase. Mol Pharmacol 59: 646-654, 2001.

25. Waiczies S, Bendix I, Prozorovski T, Ratner M, Nazarenko I, Pfueller CF, Brandt AU, Herz J, Brocke S, Ullrich O and Zipp F: Geranylgeranylation but not GTP loading determines rho migratory function in T cells. J Immunol 179: 6024-6032, 2007.

26. Kusama T, Mukai M, Tatsuta M, Nakamura $H$ and Inoue $M$ : Inhibition of transendothelial migration and invasion of human breast cancer cells by preventing geranylgeranylation of Rho. Int J Oncol 29: 217-223, 2006.

27. Jiang C, Diao F, Sang YJ, Xu N, Zhu RL, Wang XX, Chen Z, Tao WW, Yao B, Sun HX, et al: GGPP-mediated protein geranylgeranylation in oocyte is essential for the establishment of oocyte-granulosa cell communication and primary-secondary follicle transition in mouse ovary. PLoS Genet 13: e1006535, 2017.

28. Fuchs D, Berges C, Opelz G, Daniel V and Naujokat C: HMG-CoA reductase inhibitor simvastatin overcomes bortezomib-induced apoptosis resistance by disrupting a geranylgeranyl pyrophosphate-dependent survival pathway. Biochem Biophys Res Commun 374: 309-314, 2008.

29. Yokoyama K, Zimmerman K, Scholten J and Gelb MH: Differential prenyl pyrophosphate binding to mammalian protein geranylgeranyltransferase-I and protein farnesyltransferase and its consequence on the specificity of protein prenylation. J Biol Chem 272: 3944-3952, 1997.

(i) (2) This work is licensed under a Creative Commons Attribution-NonCommercial-NoDerivatives 4.0 International (CC BY-NC-ND 4.0) License. 